\title{
NORM INEQUALITIES AND CHARACTERIZATIONS OF INNER PRODUCT SPACES
}

\author{
A. Amini-Harandi, M. RAhimi And M. ReZAie
}

Abstract. Let $(X,\|\|$.$) be a real normed space and let \theta:(0, \infty) \rightarrow(0, \infty)$ be an increasing function such that $t \mapsto \frac{t}{\theta(t)}$ is non-decreasing on $(0, \infty)$. For such function, we introduce the notion of $\theta$-angular distance $\alpha_{\theta}[x, y]$, where $x, y \in X \backslash\{0\}$, and show that $X$ is an inner product space if and only if $\alpha_{\theta}[x, y] \leqslant 2 \frac{\|x-y\|}{\theta\|x\|+\theta\|y\|}$ for each $x, y \in X \backslash\{0\}$. Then, in order to generalize the Dunkl-Williams constant of $X$ [10], we introduce a new geometric constant $C_{F}(X)$ for $X$ wrt $F$, where $F:(0, \infty) \times(0, \infty) \rightarrow(0, \infty)$ is a given function, and obtain some characterizations of inner product spaces related to the constant $C_{F}(X)$. Our results generalize and extend various known results in the literature ${ }_{\text {ation }}$ (2010): 46C15, 46B20.

Keywords and phrases: Inner product space, characterizations of inner product spaces, Dunkl-Williams inequality.

\section{REFERENCES}

[1] A. M. Al-RASHed, Norm inequalities and characterizations of inner product spaces, J. Math. Anal. Appl., 176 (1993), 587-593.

[2] D. AMIR, Characterizations of Inner Product Spaces, Springer Basel, 1986.

[3] G. BIR KHOFF, Orthogonality in linear metric spaces, Duke Math., 1 (1935), 169-172.

[4] J. A. Clarkson, Uniformly convex spaces, Trans. Amer. Math. Soc., 40 (1936), 396-414.

[5] F. Dadipour And M. S. Moslehian, A characterization of inner product spaces related to the p-angular distance, J. Math. Anal. Appl., 371, 2 (2010), 677-681.

[6] M. M. DAY, Some characterizations of inner product spaces, Trans. Amer. Math. Soc., 62 (1947), $320-337$.

[7] H. DeHghan, A characterization of inner product spaces related to the skew-angular distance, Math. Notes., 93, 4 (2013), 556-560.

[8] C. F. DunKL And K. S. Williams, A simple norm inequality, Amer. Math. Monthly., 71, 1 (1964), $53-54$.

[9] R. C. JAMES, Inner products in normed linear spaces, Bull. Amer. Math. Soc., 53 (1947), 559-566.

[10] A. Jimenez-Melado, E. Llorens-Fuster and E. M. Mazcuñán-Navarro, The DunklWilliams constant, convexity, smoothness and normal structure, J. Math. Anal. Appl., 342, 1 (2008), 298-310.

[11] P. Jordan And J. von Neumann, On inner products in linear metric spaces, Ann. Math., 36, 2 (1935), 719-723.

[12] W. A. KiRK AND M. F. Smiley, Another characterization of inner product spaces, Amer. Math. Monthly., 71, 8 (1964), 890-891.

[13] E. R. LORCH, On certain implications which characterize Hilbert space, Ann. Math., 49, 3 (1948), 523-532.

[14] L. Maligranda, Simple norm inequalities, Amer. Math. Monthly., 113, 3 (2006), 256-260.

[15] H. Mizuguchi, K.-S. Saito And R. Tanaka, On the calculation of the Dunkl-Williams constant of normed linear spaces, Cent. Eur. J. Math., 11, 7 (2013), 1212-1227.

[16] R. TANAKA, T. OHWADA AND K.-S. SAITO, Geometric constants and characterizations of inner product spaces, Math. Inequal. Appl., 17, 2 (2014), 513-520. 